\title{
Enhancing the Tracking of Seedling Growth Using RGB-Depth Fusion and Deep Learning
}

\author{
Hadhami Garbouge ${ }^{1}$, Pejman Rasti ${ }^{1,2}$ and David Rousseau ${ }^{1, *}$ \\ 1 Université d'Angers, Laboratoire Angevin de Recherche en Ingénierie des Systèmes (LARIS), UMR INRAE \\ IRHS, 62 Avenue Notre Dame du Lac, 49000 Angers, France; hadhami.garbouge@univ-angers.fr (H.G.); \\ pejman.rasti@univ-angers.fr (P.R.) \\ 2 Centre d'Etudes et de Recherche pour l'Aide à la Décision (CERADE), École D'ingénieur Informatique et \\ Environnement (ESAIP), 49124 Angers, France \\ * Correspondence: david.rousseau@univ-angers.fr
}

Citation: Garbouge, H.; Rasti, P.

Rousseau, D. Enhancing the Tracking of Seedling Growth Using RGB-Depth Fusion and Deep Learning. Sensors 2021, 21, 8425. https://doi.org/10.3390/s21248425

Academic Editor: Francesco Buonamici

Received: 28 October 2021

Accepted: 14 December 2021

Published: 17 December 2021

Publisher's Note: MDPI stays neutral with regard to jurisdictional claims in published maps and institutional affiliations.

Copyright: (c) 2021 by the authors. Licensee MDPI, Basel, Switzerland. This article is an open access article distributed under the terms and conditions of the Creative Commons Attribution (CC BY) license (https:// creativecommons.org/licenses/by/ $4.0 /)$.

\begin{abstract}
The use of high-throughput phenotyping with imaging and machine learning to monitor seedling growth is a tough yet intriguing subject in plant research. This has been recently addressed with low-cost RGB imaging sensors and deep learning during day time. RGB-Depth imaging devices are also accessible at low-cost and this opens opportunities to extend the monitoring of seedling during days and nights. In this article, we investigate the added value to fuse RGB imaging with depth imaging for this task of seedling growth stage monitoring. We propose a deep learning architecture along with RGB-Depth fusion to categorize the three first stages of seedling growth. Results show an average performance improvement of $5 \%$ correct recognition rate by comparison with the sole use of RGB images during the day. The best performances are obtained with the early fusion of RGB and Depth. Also, Depth is shown to enable the detection of growth stage in the absence of the light.
\end{abstract}

Keywords: deep learning; plant growth; CNN; RGB-Depth; image fusion; feature fusion; transformers

\section{Introduction}

The detection of the seedling growth stages is a fundamental problem in plant science. This covers the emergence of seedling from the soil, the opening of cotyledons and appearance of the first leave which correspond to the earliest stages of development of plant. The success or failure of these developmental stages and their kinetics have a huge impact on the evolution of the future plant. Recently, seedling growth monitoring has received attention from the computer vision community [1-16]. Among these works, the state-of-the-art approach based on deep learning proposed in [16] has shown the possibility to automatically classify the stages of development of seedling with RGB sequences of images from top view with an accuracy higher than $90 \%$.

One of the limitations of the work proposed in [16] is that the monitoring was done only during daylight with RGB images. Consequently, any events happening during the night would be missed and/or possibly estimated with a temporal bias. In this article, we propose an extension of the work of [16] and investigate the possibility to push forward the monitoring of the seedling growth during the day and the night. To this purpose, RGB-Depth camera were used. These technologies have been demonstrated of wide value in plant phenotyping [17-24]. The depth images are computed by an active LIDAR camera operating in infrared (IR). This camera can be activated during day and night without impact on the development of the plants. As in [16] we selected low-cost versions of these RGB-Depth cameras. These low-cost constraints are specially important in plant phenotyping [25] when moving the plants or the camera is not an option and that replication of cohorts of cameras is to be chosen to monitor large populations of plants. Low-cost RGB-Depth cameras are also logically coming with artifacts and noise. Such artifacts and 
metrological limitations of low-cost RGB-Depth cameras have been extensively studied (see [26] for a recent survey). In our case, we rather work at an informational level. We focus on a classification task, i.e., a nonlinear decision, which is by nature more robust to noise since it does not have to provide a high-fidelity, metrological, linear estimation. The hypothesis investigated in this article is that these low-cost RGB-Depth sensors despite their limited spatial resolution and the presence of artifacts may be of enough value to enhance the tracking of seedling growth during day and night.

We demonstrate, for the first time, to the best of our knowledge the value of these RGB-Depth images to monitor the early stages of seedling growth. We investigate fusion strategies between RGB and depth with several neural networks architecture. The underlying motivation to use multimodal data is that complementary information give a richer representation that may be utilized to create better results than a single modality. The multimodal fusion research community has made significant progress in the past decade [27]. Different fusion strategies have been reviewed [28,29]. Specifically for RGB and Depth with deep learning architectures, fusion has been extensively studied in the literature [30-41]. Mainly two types of fusion can be distinguished. First, images can be stacked at the input: this is the early fusion [30-34], that we call image fusion. Second, deep features can be independently extracted and then fused before a classification stage: this is the feature fusion [35-38]. In this work, we investigate these fusions scenarios that we applied to the important problem of seedling growth stage monitoring. Since we process sequences of images we considered time-dependent neural network architectures. As in [16], we included a base line convolutional neural network (CNN) and LSTM [42]. We also added TD-CNN GRU [43] and transformer [44] which were not included in [16].

\section{Materials and Methods}

\subsection{Imaging System and Data Set}

We have conducted similar experiments as the ones described in detail in [16] and shortly recalled here. A set of minicomputers, connected to RGB-Depth cameras [45], was used to image seedlings from the top view as illustrated in Figure 1. We used, instead of the RGB cameras of [16], Intel real sense cameras [46] (model D435) which natively produces registered RGB-Depth pairs of images and calibrated Depth maps. We installed 8 of these RGB-Depth cameras in a growth chamber where cameras followed the growth of seedlings from top view. During experiment, soil pots were hydrated to saturation for $24 \mathrm{~h}$ after which excess water was removed. After $24 \mathrm{~h}$, seeds were sown at a depth of $2 \mathrm{~cm}$, and trays were placed in a growth chamber at $20^{\circ} \mathrm{C} / 16^{\circ} \mathrm{C}$, with $16 \mathrm{~h}$ for photoperiod at $200 \mu \mathrm{Mm}^{-2} \mathrm{~s}^{-2}$. The soil was kept wet throughout the experiments. Each experiment took one week with a frame rate of $15 \mathrm{~min}$. The time lapse program (made in Python) was implemented on a central minicomputer controlling, via ethernet wires, the 8 minicomputers connected to the RGB-Depth cameras.

Concerning the biological material, seedling growth was recorded for 2 experiments using seed lots from different accessions of beans such as Flavert, Red Hawk, Linex, Caprice, Deezer and Vanilla. Each experiment consisted of 3 trays with 40 pots in which 120 seeds of accessions were sown. There is a similarity between the species in this experiment and the two species which were used in [16] as all of them consist in dicotyledon species. The main difference between them comes from the number of varieties in this experiment which is three times higher than the one in [16].

In total, the database consists of 72 temporal sequences of RGB and depth images of size $66 \times 66$ pixels where each temporal sequence consists of 616 individual images. Example of images from the database is shown in Figure 1. RGB-Depth temporal sequences acquired during daylight were annotated by expert in biology while looking at RGB images. This ground-truth annotation consisted of four classes: soil, first appearance of the cotyledon (FA), opening of the cotyledon (OC), and appearance of the first leaf (FL). The algorithms presented in this paper for seedling emergence identification following these four phases of growth were trained, validated, and tested against this human-annotated 
ground-truth. In order to train robust models, we used the cross-validation approach by considering image sequences of bean varieties in three split of train, validation, and test dataset. Table 1 provides a synthetic view of the data set used for training and testing of the models. For the training dataset, we applied data augmentation using a simple horizontal flip on each temporal sequence.

Table 1. Description of the RGB-Depth dataset used in this study.

\begin{tabular}{|c|c|c|c|c|c|}
\hline & Species & $\begin{array}{l}\text { No. of Temporal } \\
\text { Sequences }\end{array}$ & $\begin{array}{c}\text { Totale No. of Images } \\
\text { during Days }\end{array}$ & $\begin{array}{c}\text { Totale No. of Images } \\
\text { during Nights }\end{array}$ & $\begin{array}{l}\text { Totale No. of All } \\
\text { Images }\end{array}$ \\
\hline \multirow{6}{*}{ Training dataset } & Flavert & 10 & 4240 & 1920 & \multirow{6}{*}{36,960} \\
\hline & Red Hawk & 10 & 4240 & 1920 & \\
\hline & Linex & 10 & 4240 & 1920 & \\
\hline & Caprice & 10 & 4240 & 1920 & \\
\hline & Deezer & 10 & 4240 & 1920 & \\
\hline & Vanilla & 10 & 4240 & 1920 & \\
\hline \multirow{6}{*}{ Validation dataset } & Flavert & 1 & 424 & 192 & \multirow{6}{*}{3696} \\
\hline & Red Hawk & 1 & 424 & 192 & \\
\hline & Linex & 1 & 424 & 192 & \\
\hline & Caprice & 1 & 424 & 192 & \\
\hline & Deezer & 1 & 424 & 192 & \\
\hline & Vanilla & 1 & 424 & 192 & \\
\hline \multirow{6}{*}{ Testing dataset } & Flavert & 1 & 424 & 192 & \multirow{6}{*}{3696} \\
\hline & Red Hawk & 1 & 424 & 192 & \\
\hline & Linex & 1 & 424 & 192 & \\
\hline & Caprice & 1 & 424 & 192 & \\
\hline & Deezer & 1 & 424 & 192 & \\
\hline & Vanilla & 1 & 424 & 192 & \\
\hline
\end{tabular}

Depth images can contain artifacts with missing values. This can happen on part of the scene where not enough light is reflected or for objects that are too close or too far from the camera. While neural networks should be able to cope with such noise, it is better to correct them to use the capability of these networks on clean data. In order to correct these artifacts, we applied a classical inpainting technique [47] of depth images to reduce the noise.
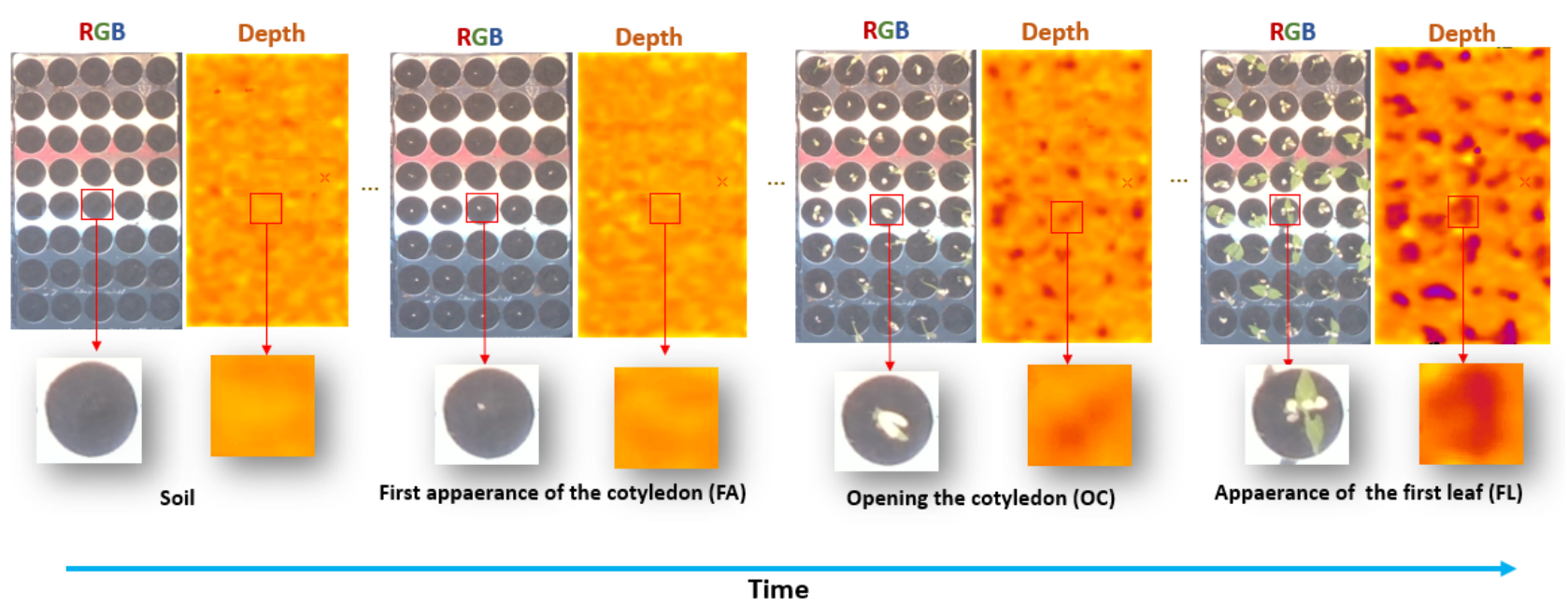

Figure 1. Overview of the time-lapse collected for this work. Upper row, view of a full tray with 72 pots from top view. Lower row, a zoom on a single pot at each stage of development to be detected from left to right: soil, first appearance of the cotyledon (FA), opening the cotyledons (OC) and appearance of the first leaf (FL). 


\subsection{RGB-Depth Deep Learning Fusion Strategies}

We describe here the different neural network architectures tested in this study to fuse the RGB and Depth for the classification of seedling growth stages as depicted in Figure 2.

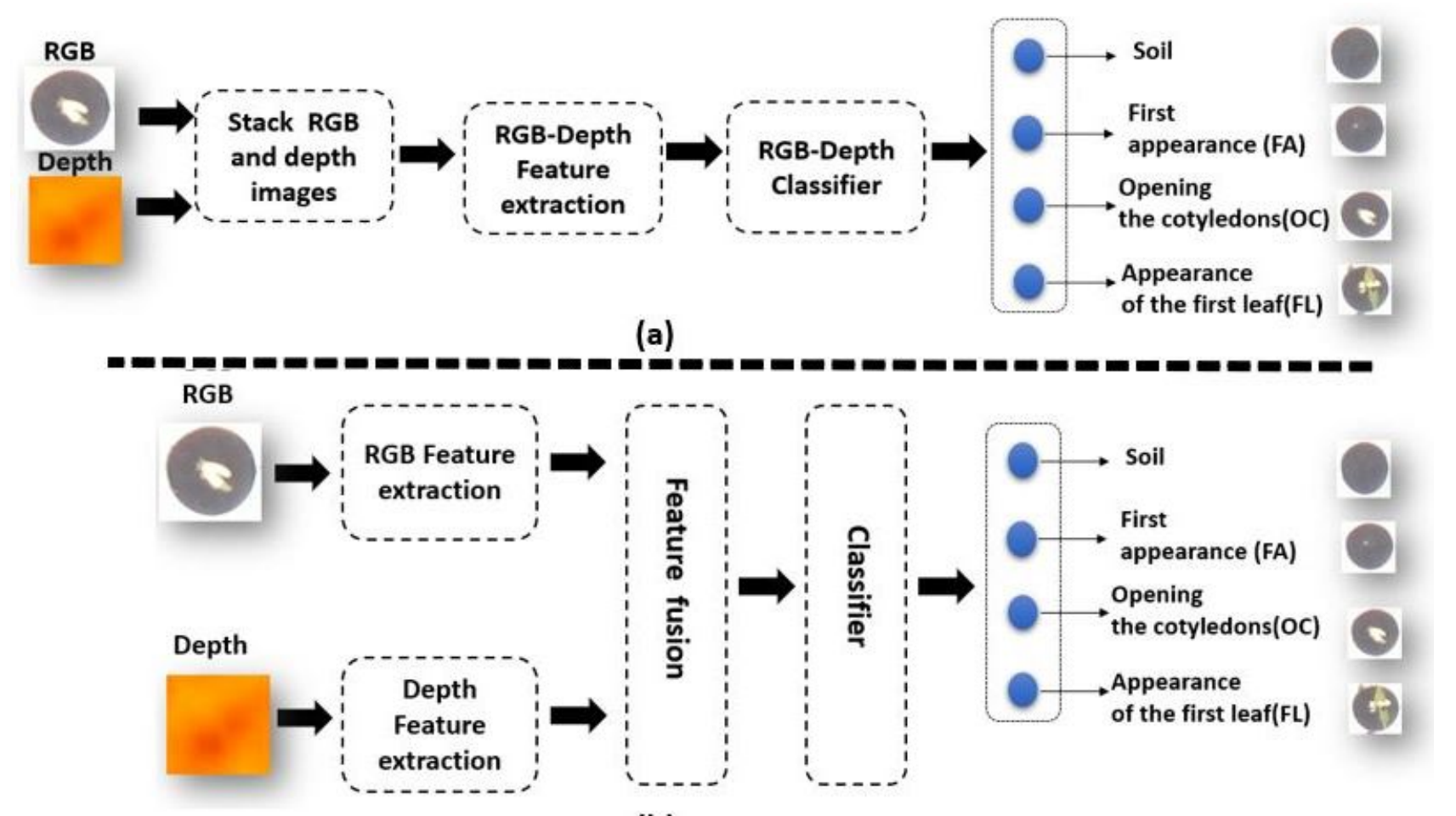

(b)

Figure 2. Different types of RGB-Depth fusion architectures tested in this article for image classification. (a) Image-based RGB-Depth fusion, (b) Feature-based RGB-Depth fusion.

\subsubsection{CNN-Based Image Early Fusion Learning Structure}

We first integrated, as in [48], RGB and Depth data stacked in a four-channel as input to a CNN (see Figure 3a). The feature extraction block from four-channel input images is followed by the classification block (shown in Figure 3a). The CNN architecture is the one of $[16,43]$ that we shortly recall. The feature extraction block of a CNN model is responsible for extracting features from input images using convolutional layers, whereas the classification block determines classes. To keep the amount of train parameters low, we created an AlexNet [49] like CNN structure. This architecture reads as follows: four convolutional layers with filters of size $3 \times 3$ and respective numbers of filters 64,128 , 256, and 256 each followed by rectified linear unit (RelU) activations and $2 \times 2$ maxpooling; a fully connected layer with 512 units, ReLU activation and dropout $(p=0.5)$ and a fully connected output layer for four classes corresponding to each event with a softmax activation. This proposed CNN architecture has been optimized on a hold-out set and was demonstrated in [16] to be optimal by comparison with other standard classical architectures (VGG16, ResNet, DenseNet). The network was trained from scratch since the size of the input tensor (4 channels and small spatial resolution) was different from existing pre-trained networks on large RGB data sets. 


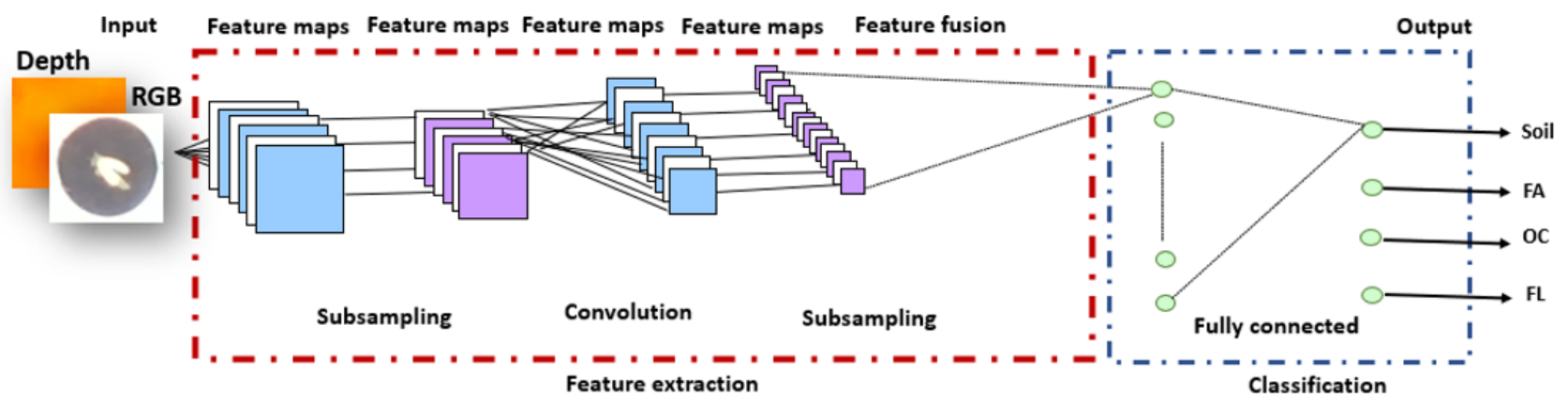

(a)

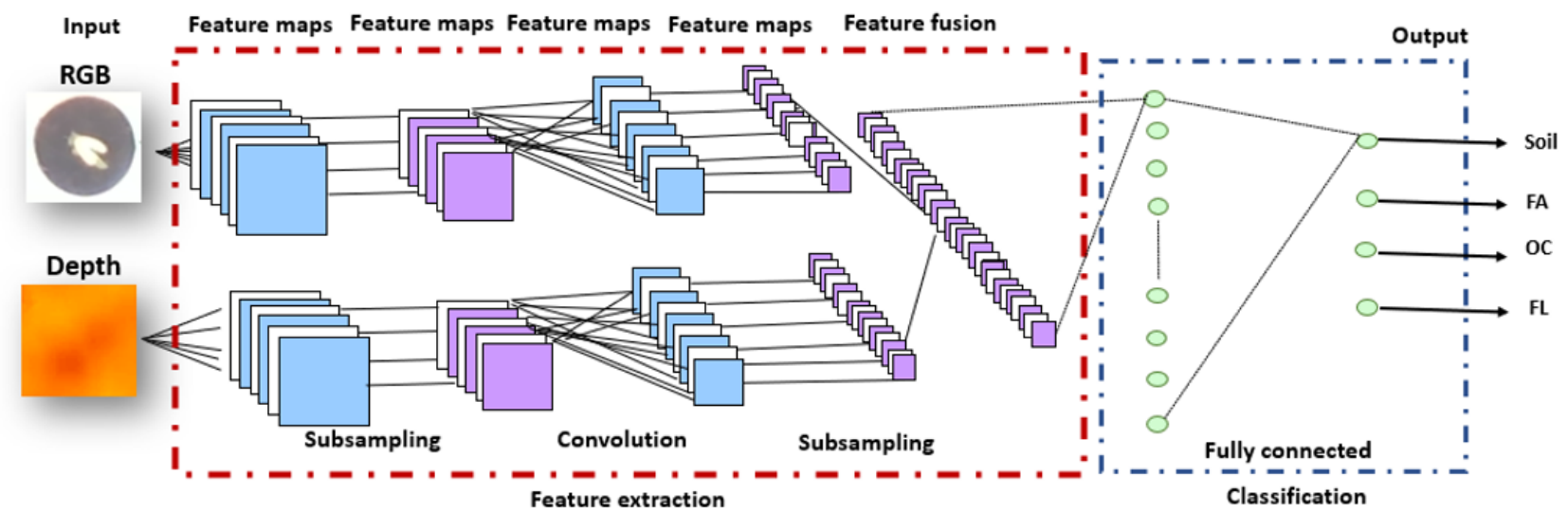

(b)

Figure 3. (a) CNN architecture of image fusion for RGB-Depth, (b) CNN architecture of features fusion for RGB-Depth.

\subsubsection{CNN-Based Feature Fusion Learning Structure}

Our architecture, shown in Figure 3b, is made up of two convolutional network streams that operate on RGB and Depth data, respectively. The same structure of image fusion $\mathrm{CNN}$ has been developed for each stream of the feature fusion CNN. The feature extractor part of the CNN architectures of RGB and Depth images consists of four convolutional layers which have 64, 128, 256, and 256 filters, respectively (similar to the AlexNet like structure of the previous subsection). The ReLU activation function is considered for each convolutional layer followed by a max-pooling layer. On the classification part of the $\mathrm{CNN}$ architectures, a fully connected layer with 512 units, and an output layer with four neurons corresponding to each event with a softmax activation function.

\subsubsection{TD-CNN-GRU-Based Image and Feature Fusion Learning Structure}

We demonstrated in $[16,43]$ the possible added value to embed in controlled environment a memory in the process of the sequence of images. We demonstrated in [43], the superiority of Time dependent CNN with gated recurrent units (TD-CNN-GRU) by comparison with other memory based methods such as long short term memory (LSTM) and CNN-LSTM architectures. GRU uses two gates: the update gate and the reset gate while there are three gates in LSTM. This difference makes GRU faster to train and with better performance than LSTMs on less training data [50]. The same CNN architecture of our model in [16] was embedded in our TD-CNN-GRU model where the optimal duration of the memory was found to be 4 images in $[16,43]$ corresponding to 1 hour of recording. Figure 4 shows a schematic view of the proposed TD-CNN-GRU for images and feature fusion respectively. 

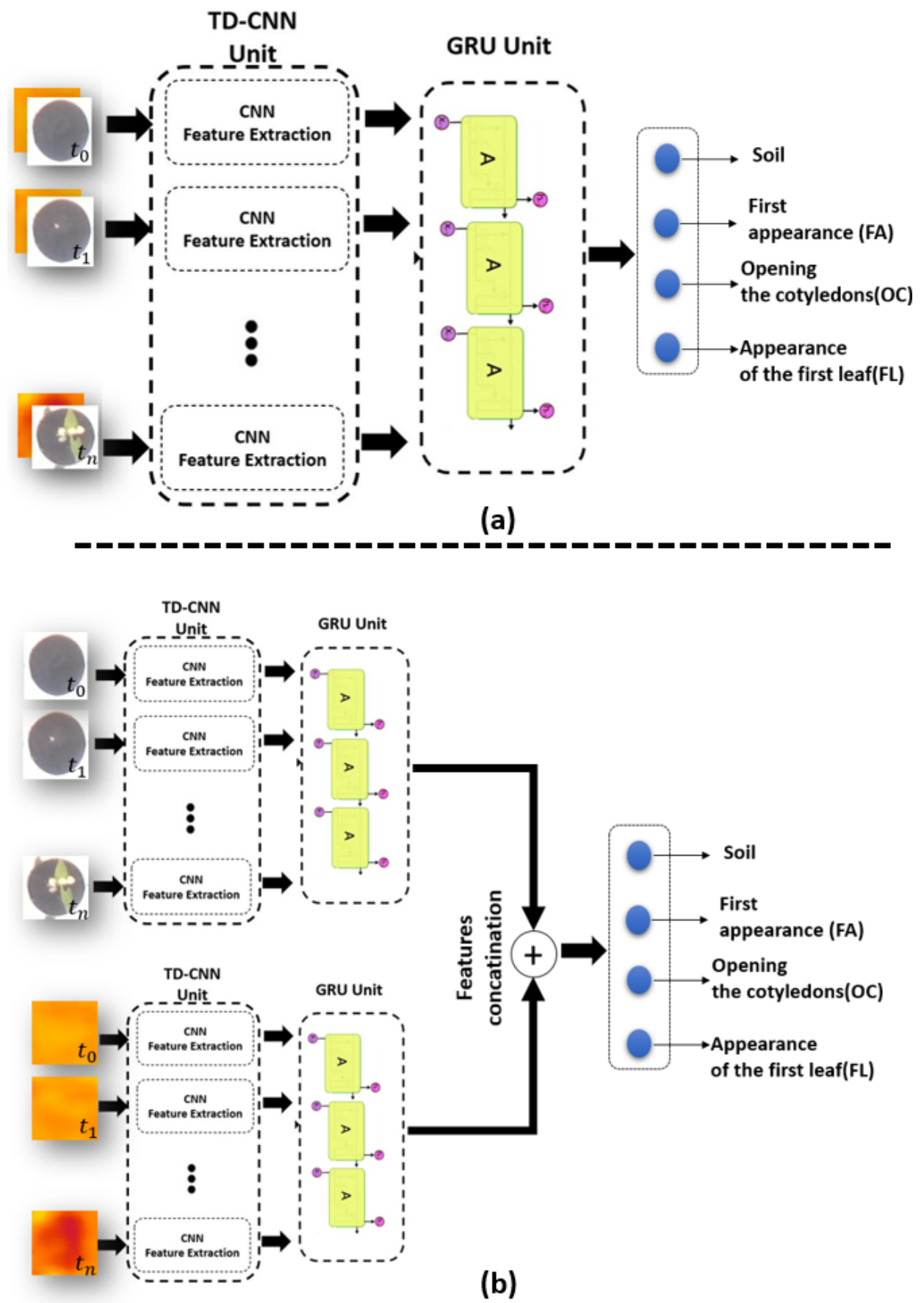

Figure 4. (a) TD-CNN-GRU architecture of image fusion for RGB-Depth, (b) TD-CNN-GRU architecture of features fusion for RGB-Depth.

\subsubsection{Transformers-Based Image and Feature Fusion Learning Structure}

A last class of neural network dedicated to time series are the transformers. Since their introduction in [44] they have been shown to outperform recurrent neural networks such as LSTM and GRU specially in the field of natural language processing as they do not require that the sequential data be processed in order. Transformers have been shown suitable to process temporal information carried by single pixels in satellite images time series [51-53]. Transformers have recently been extended to the process of images [54] where images were analysed as a mosaic of subparts of the original images creating artificial time series. In our case, we directly have meaningful original images which corresponds to the field of view of the pots. We, therefore, provide the transformer of [54] with time 
series of consecutive images of the same pot (we used the same time slot as in the other spatio-temporal methods). We used 32 transformer layers with batch size 64, feed forward layer as classification head layer and the size of our patch size was equal to $66 \times 66$ pixels for both architectures of Figure 5.
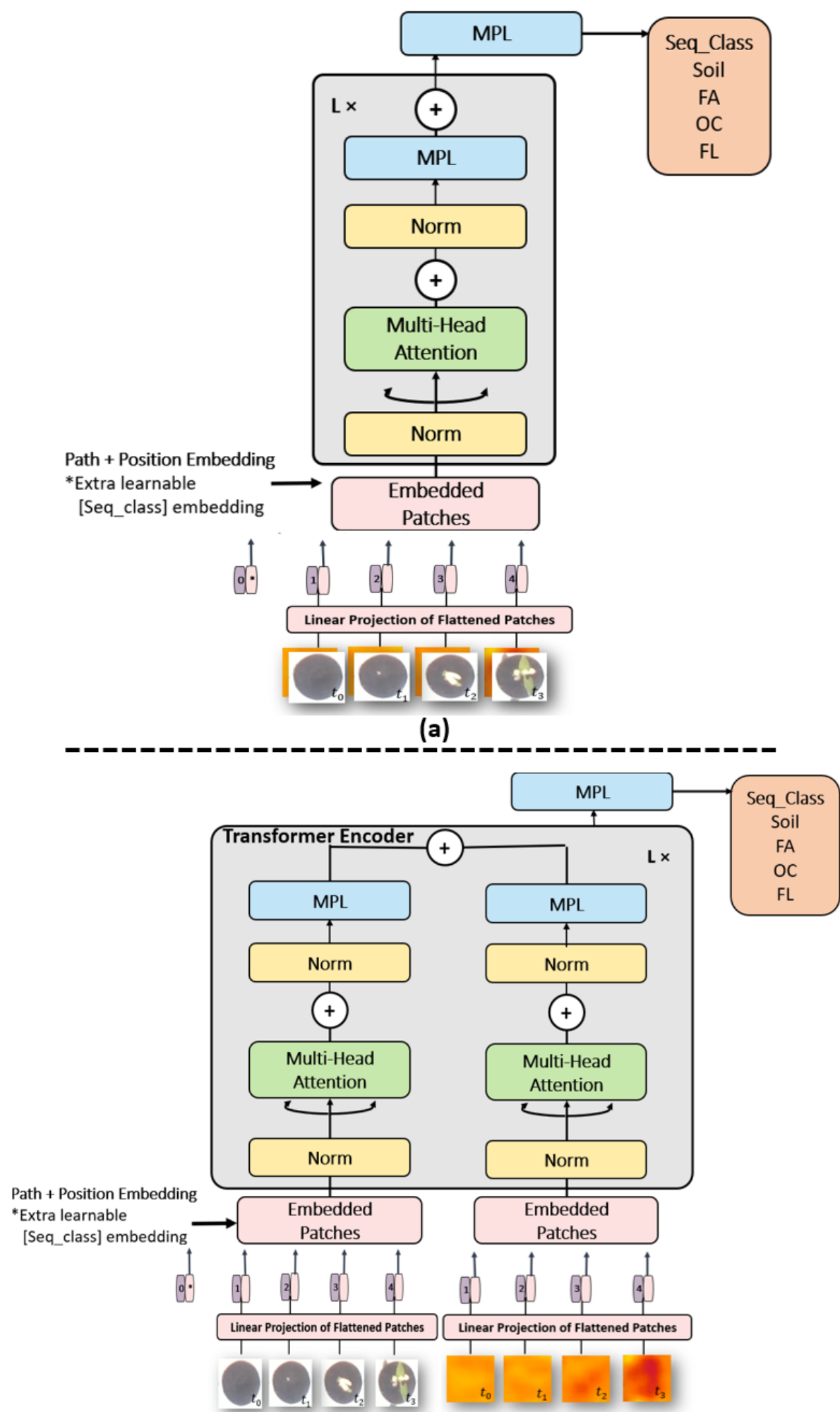

(b)

Figure 5. (a) Transformer architecture of image fusion for RGB-Depth, (b) Transformer architecture of features fusion for RGB-Depth. 
For all our training, we used the NVDIA DGX station. This station is composed of 4 GPUs and each one of them have a RAM memory of $32 \mathrm{~Gb}$. We used Python version 3.7.8, Tensor-flow version 2.7.0 and Keras library version 2.3.1.

\subsection{Accuracy}

The performances of the different fusion strategies tested on our dataset were classically assessed with Accuracy

$$
\text { Accuracy }=\frac{T P+T N}{T P+T N+F P+F N},
$$

where TP, TN, FP, and FN stands for true positive, true negative, false positive, and false negative).

\section{Results}

\subsection{Fusion Strategies}

The proposed deep learning methods CNN, TD-CNN-GRU, and Transformers with image or feature RGB-Depth fusion were applied to the produced dataset as described in the Section 2. The performances are provided in Tables 2-4 and Figure 6.

Table 2. Seedling growth stage classification average accuracy and standard deviation when performed over 10 repetitions of $\mathrm{CNN}$ model.

\begin{tabular}{lccc}
\hline & Training & Validation & Test \\
\hline RGB & $0.95 \pm 0.02$ & $0.91 \pm 0.03$ & $0.88 \pm 0.05$ \\
\hline Image fusion RGB-Depth & $0.97 \pm 0.02$ & $0.95 \pm 0.02$ & $0.94 \pm 0.04$ \\
\hline Features fusion RGB-Depth & $0.97 \pm 0.01$ & $0.96 \pm 0.01$ & $0.94 \pm 0.01$ \\
\hline
\end{tabular}

Table 3. Seedling growth stage classification average accuracy and standard deviation when performed over 10 repetitions of TD-CNN-GRU model.

\begin{tabular}{lclc}
\hline & Training & Validation & Test \\
\hline RGB & $0.87 \pm 0.02$ & $0.85 \pm 0.01$ & $0.80 \pm 0.01$ \\
\hline Image fusion RGB-Depth & $0.91 \pm 0.01$ & $0.87 \pm 0.02$ & $0.82 \pm 0.01$ \\
\hline Features fusion RGB-Depth & $0.90 \pm 0.01$ & $0.86 \pm 0.02$ & $0.81 \pm 0.01$ \\
\hline
\end{tabular}

Table 4. Seedling growth stage classification average accuracy and standard deviation when performed over 10 repetitions of transformer model.

\begin{tabular}{lccc}
\hline & Training & Validation & Test \\
\hline RGB & $0.90 \pm 0.02$ & $0.86 \pm 0.01$ & $0.82 \pm 0.01$ \\
\hline Image fusion RGB-Depth & $0.96 \pm 0.02$ & $0.91 \pm 0.01$ & $0.88 \pm 0.03$ \\
\hline Features fusion RGB-Depth & $0.92 \pm 0.03$ & $0.89 \pm 0.02$ & $0.84 \pm 0.01$ \\
\hline
\end{tabular}

Tables 2-4 show that all methods performed better when RGB and Depth data are fused by comparison with the sole use of RGB data. This improvement is obtained both with image fusion and with feature fusion. This demonstrate the value of RGB-Depth fusion with a gain of $5 \%$ (on average) compared to the use of the sole RGB images. This is obtained at a reasonable training time of around 1 to $3 \mathrm{~h}$ as detailed in Table 5 . The best results are obtained with the $\mathrm{CNN}$ method, i.e., the spatial method by comparison with the spatio-temporal method. This $\mathrm{CNN}$ is showing the best absolute performance, the smallest training time and also minimum decrease of performance between training, validation and test. This is in agreement with our previous results found in [16,43], where spatio-temporal 
methods outperformed memoryless spatial ones only when the kinetic of growth were homogeneous among the dataset. This was not the case in this study.

Table 5. Training time of the different deep learning architectures.

\begin{tabular}{ccc}
\hline & \multicolumn{1}{c}{ Model } & Training Time \\
\hline \multirow{2}{*}{ RGB } & CNN & $1 \mathrm{~h} 00 \mathrm{~min}$ \\
\cline { 2 - 3 } & Transformer & $1 \mathrm{~h} 30 \mathrm{~min}$ \\
\cline { 2 - 3 } Image fusion RGB-Depth & TD-CNN-GRU & $3 \mathrm{~h} 00 \mathrm{~min}$ \\
\cline { 2 - 3 } & CNN & $1 \mathrm{~h} 15 \mathrm{~min}$ \\
\cline { 2 - 3 } & Transformer & $1 \mathrm{~h} 35 \mathrm{~min}$ \\
\hline \multirow{3}{*}{ Features fusion RGB-Depth } & TD-CNN-GRU & $3 \mathrm{~h} 30 \mathrm{~min}$ \\
\cline { 2 - 3 } & CNN & $1 \mathrm{~h} 20 \mathrm{~min}$ \\
\cline { 2 - 3 } & Transformer & $1 \mathrm{~h} 30 \mathrm{~min}$ \\
\hline
\end{tabular}

The confusion matrix of the CNN method is displayed in Figure 6 for RGB images and RGB-Depth images. Interestingly errors with both RGB and RGB-Depth only occur on adjacent classes along the developmental order. These are situations where even the human eye can have uncertainty to decide the exact time of switching from one class to the next one. Remaining errors can thus be considered as reasonable errors. The confusion matrices also clearly demonstrate that the main gain brought by the Depth channel is on the stage of opening the cotyledons for which the error are divided by a factor two. First appearance out of the soil, or the appearance of the first leave produce very limited variations on the depth. By contrast, the opening of the cotyledons produces an abrupt variation of the Depth. Therefore, the impact of Depth on the improvement of the performance of classification on this developmental stage is consistent with this rationale. Following also this rationale, one can notice that the errors on opening the cotyledon slightly increase when Depth is added but the overall impact of Depth is on average beneficial to the global accuracy.

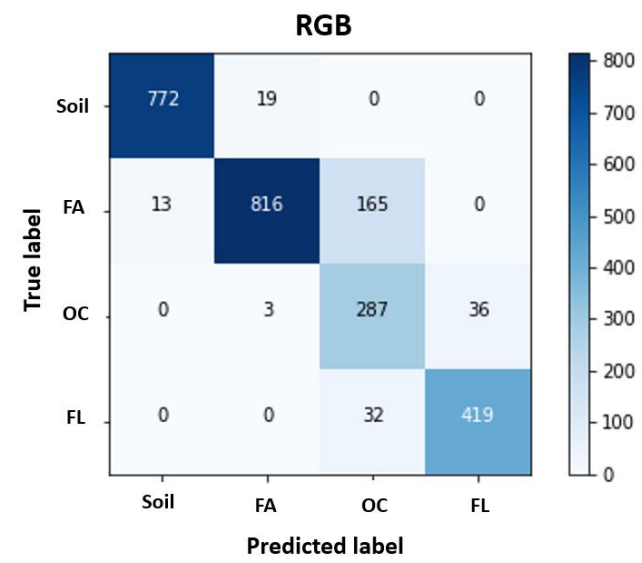

(a)

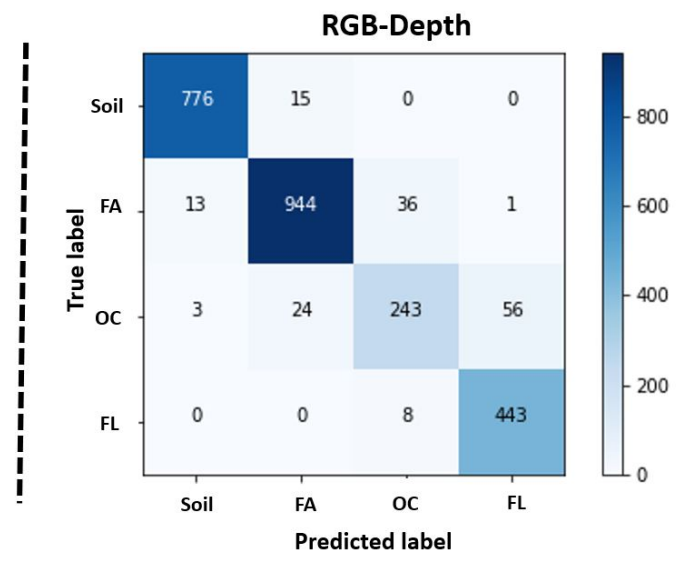

(b)

Figure 6. Confusion matrices for the best method found in Table 2, i.e., CNN. (a) for the RGB images and (b) for the RGB-Depth images.

\subsection{Detection of Event Changes at Night Using Depth Information}

The advantage of using the depth is not limited to enhance the performance during the day as shown in the previous subsection. Depth is also expected to be specifically useful during the night since the RGB cameras are then non operating while the Depth 
images can still be acquired. If the growth stage switches during the night the RGB imaging devices detect the switch only on the first frame of the next day time as illustrated in Figure 7. It is possible to screen for Depth alone during these nights and observe the start of a growth pattern actually occurring before the beginning of the day. We demonstrate in this subsection how to take benefit quantitatively of the sole Depth channel during these nights.

We analyzed the number of switches from one growth stage to another happening on the first image acquired during the day in the data set of [16] and found out that it represented 35 percent of the events (see Figure 8). This is similar to what we found with the dataset of this article where we had 100 sets of pots from different varieties. In these frames, we have 115 switches of growth stages with 43 happening during night time. While some could be triggered by the action of light others could also happen earlier during the night. To detect a possible change during the night, we quantitatively used Depth. We designed Algorithm 1 which acts as follows. We first detects nights where a switch between a growth stage to another growth stage is found in RGB images. During these nights, the algorithm then detects the depth frame on which the switch is the most likely to occur. In short, this is obtained by choosing the time where the average spatial depth is permanently (computed over a sliding window of 4 images $=1 \mathrm{~h}$ ) closer to the average spatial depth of the next growth stage.
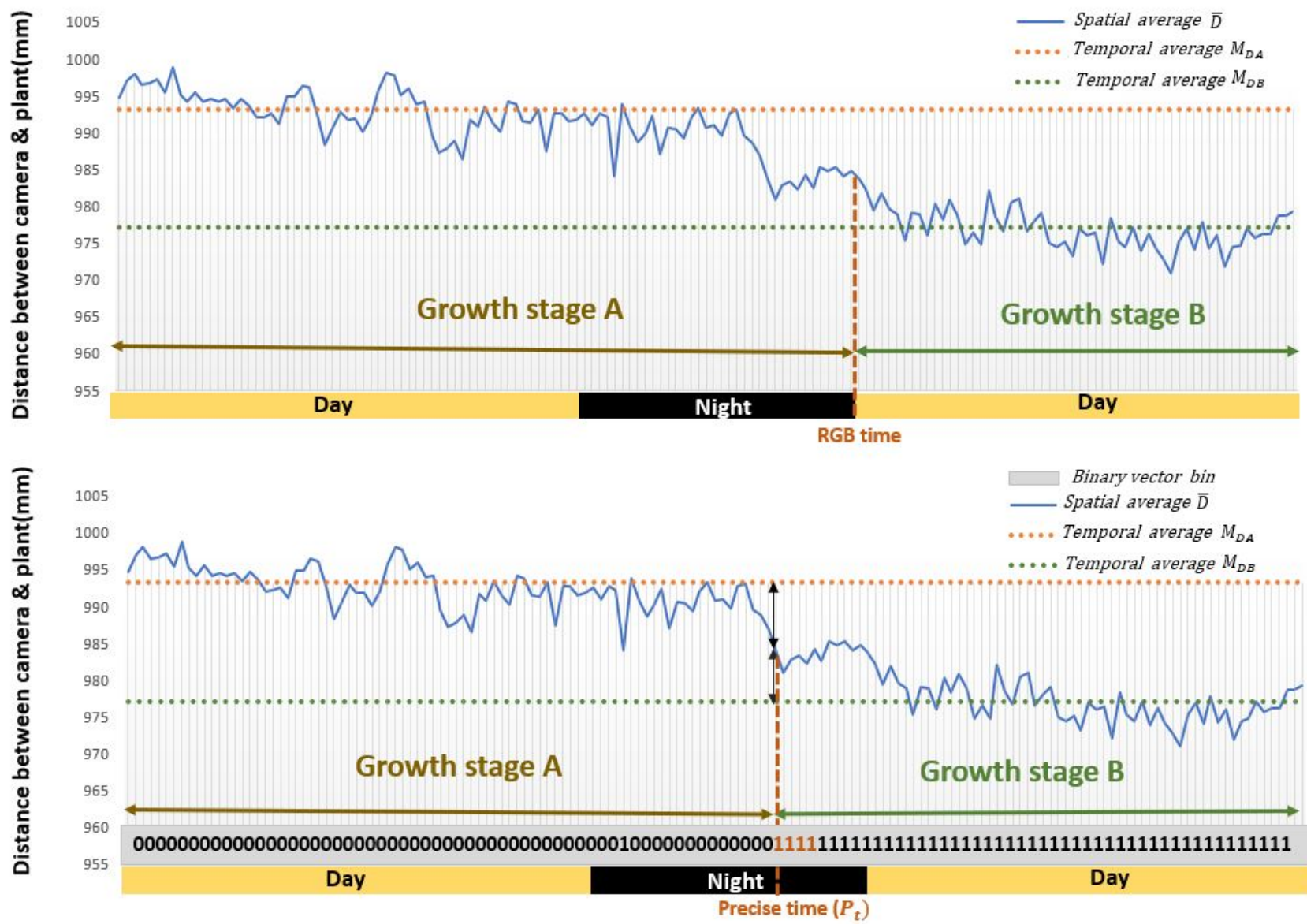

Figure 7. First row: the detection of switch from growth stage A to growth stage B using only daytime RGB images. Second row: the more precise detection of switch from growth stage A to growth stage B using the Depth pattern during the night time as proposed by Algorithm 1. 


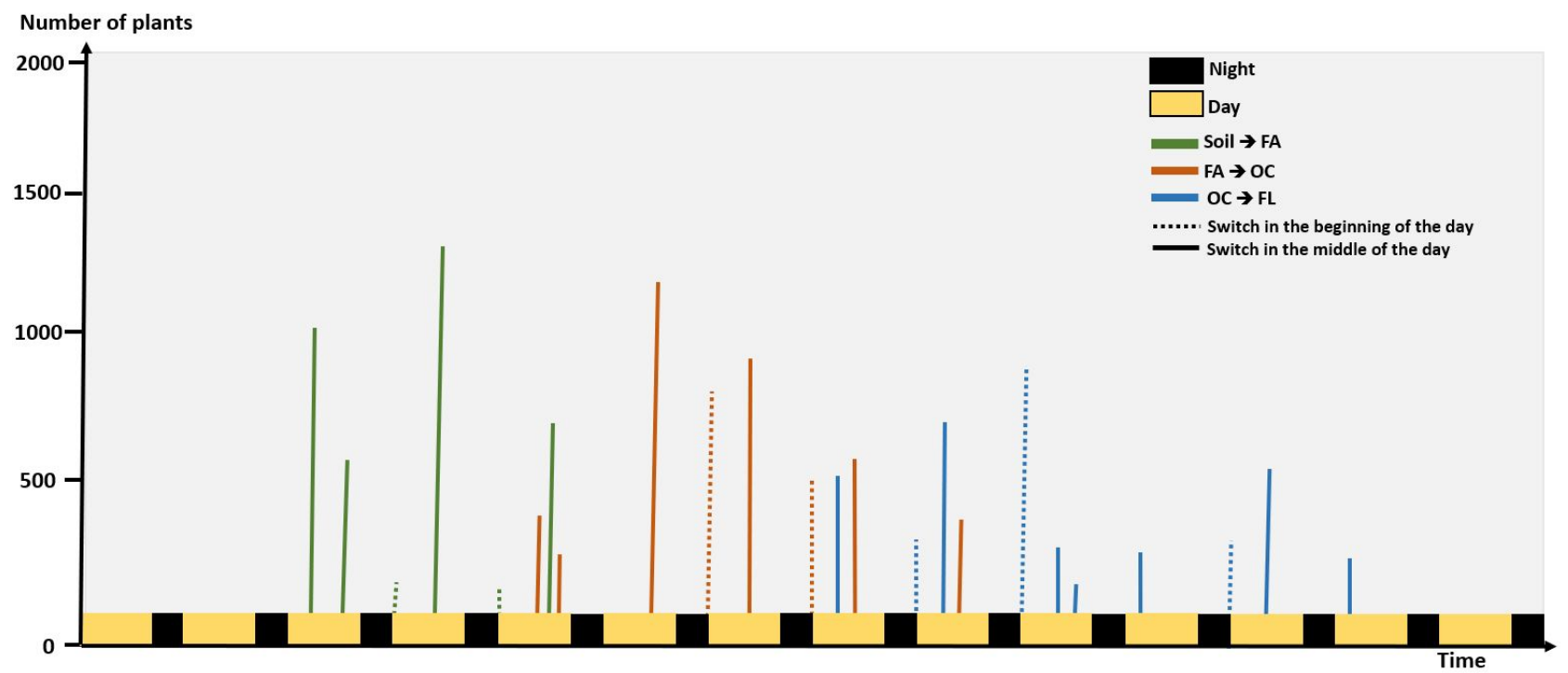

Figure 8. Histogram of detection of growth stage change during day and night from 4000 plants.

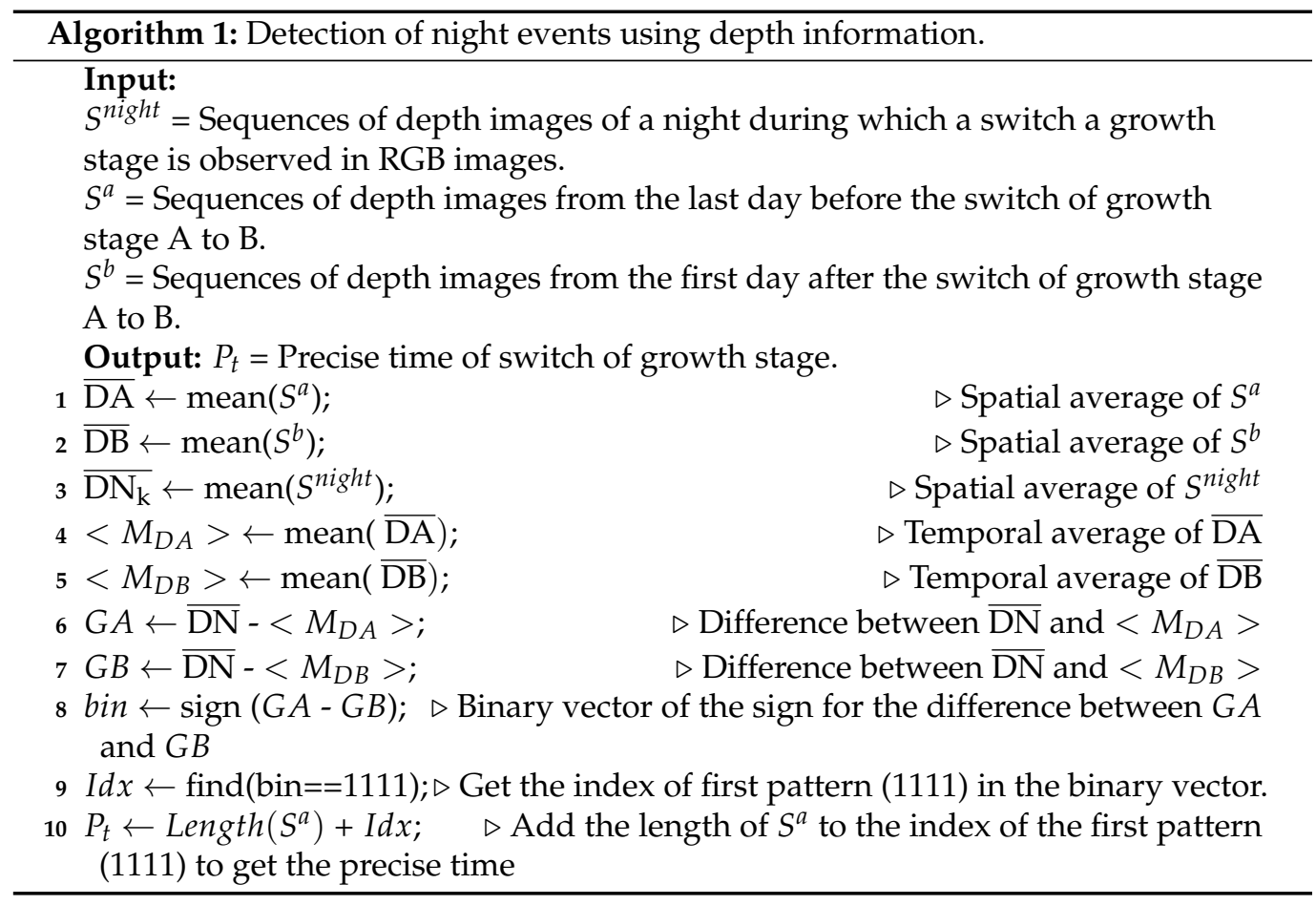

To validate Algorithm 1, we could not establish ground truth during the night. As a workaround, we used daylight events and applied the depth channel only to the Algorithm 1. Then, we used the annotated ground truth obtained from the RGB images to compute the performance of Algorithm 1. We found $80 \%$ of these 115 switches with a shift of less than 4 frames on average (standard deviation of 2 frames) by comparison with the manually annotated ground truth. This corresponds to an uncertainty (bias here) of $1 \mathrm{~h}$ which is very reasonable and much lower than the error duration of the night itself $(8 \mathrm{~h})$ if no Depth were used. 


\section{Discussion}

We analyzed the remaining errors of the proposed algorithms and discuss them in this section together with some open perspectives of the work.

Two main sources of errors can be attached to the acquisition protocol and instrumentation itself. These are illustrated in Figure 9. First, some seedlings growth so fast that their leaves or cotyledons go out of the observation window (Figure 9a). This causes drop in depth and change in the RGB pattern. With our current approach, we do focus on individual pots. For such seedlings growing at early stages outside of their pot, we would need to either use larger pots or develop tracking algorithms. This falls outside of the scope of this study which focused on the added value of Depth when fused to RGB for the detection of early growth stages of seedlings. Another source of errors happens due to noise on the Depth channel (Figure 9b). Such noises were observed when too much or too low amount of IR light was reflected on pots. This happens for instance when the plastic material of the pots has a high reflectance or when some remaining water(absorbing IR) is present. These noises can be reduced by carefully choosing the material used for the pot and the watering process. Another type of error comes from the inherent large heterogeneity of shapes and sizes of the bean varieties considered in this study and illustrated in Figure 10. This affects specially the detection of growth stage which shows the tiniest changes, i.e., the opening of the cotyledons. To solve these errors, one could simply add more data or use more advanced data augmentation techniques such as zoom, stretch, color jitter, ... We wanted to provide basic results here which already happen to be of rather high quality without the use of such approach to robustify the model since the main goal was the fusion of the RGB and Depth for seedling growth monitoring.
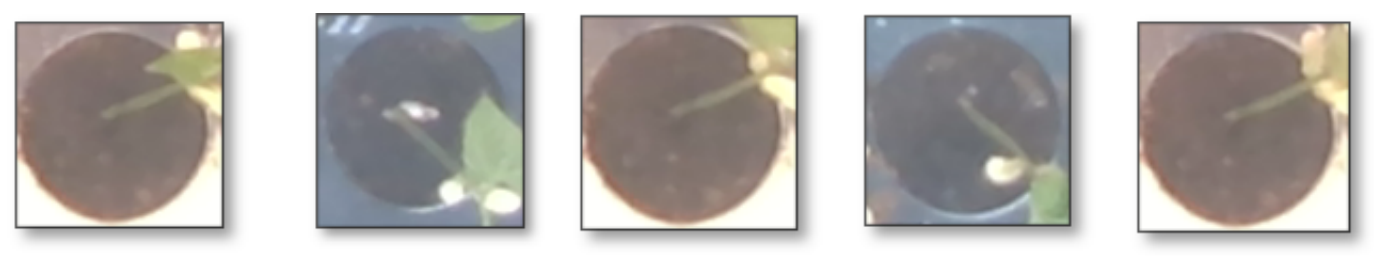

\section{(a)}
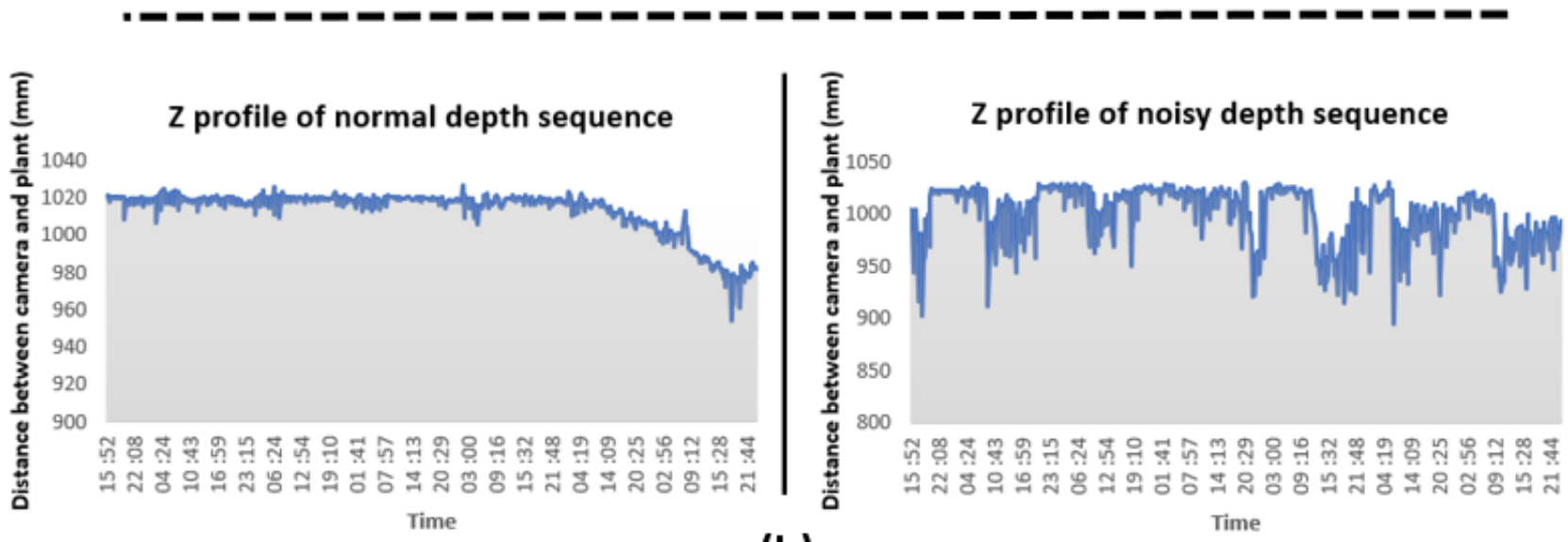

(b)

Figure 9. Sources of errors due to the acquisition protocol (a) and instrumentation (b). 


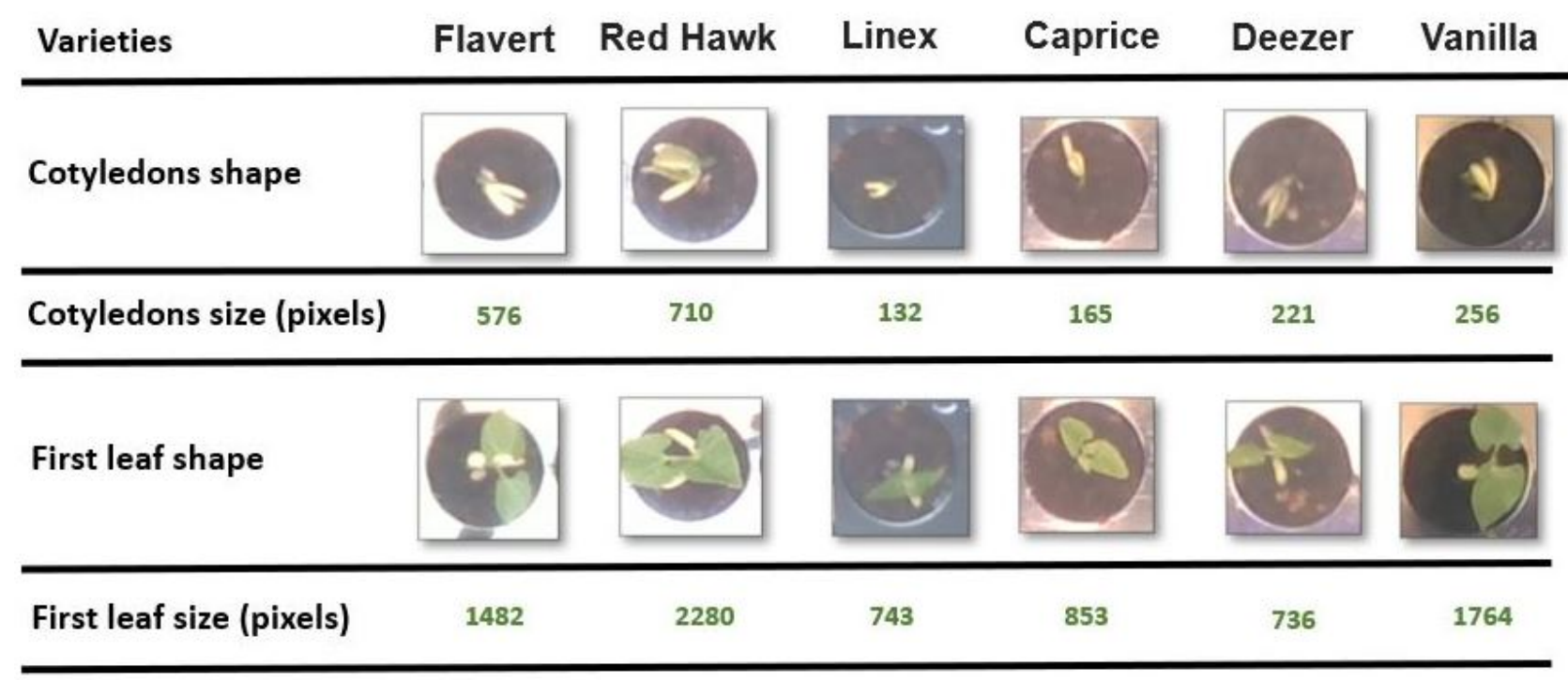

Figure 10. Heterogeneity of shape and size in the two events OC and FL for the different bean varieties used in the training.

One may wonder about the robustness of the model proposed given the relatively small size of the plant population considered. First, the overfit measured with the best method was found to be limited together with the difference of performance between cultivars. It is important to recall here that the point of the work is to quantify the added value of RGB-Depth images by comparison with sole RGB. This is what we do on the same data sets. Interestingly, the performance with RGB images obtained with only 72 samples are similar to the larger data set used in [16] (90\% against $88 \%$ here). However, we cannot ensure a perfect robustness to large change of phenotypic shapes. If such variability in scale was expected, larger data sets would have to be constituted. The comparison between RGB and RGB-Depth would remain unchanged.

In this work, we focused on early fusion and feature fusion of RGB and Depth. One may also consider decision fusion where the classification from the RGB image and the Depth image would be made. We performed this analysis and found a pure random decision when the classification was made on Depth alone. Therefore, at the decision level, no added value of Depth was to be expected on average. Fusion between RGB and Depth for such small images and low-cost sensors as the one considered in this study is found to be beneficial on average at earlier stages of processing (image or features). However, after analysing the confusion matrix in detail, one could imagine to selectively using the added value of Depth at the stages of growth where it is expected to be the most significant. This was found to be between the FA and OC in our case and more generally when large contrast in Depth happens. On the contrary, one could discard the use of Depth when the growth process is estimated to lay at stages where no contrast in Depth is expected (between Soil and FA in our case).

This work could be developed in several other future directions. First, we could revisit this study with higher resolution Depth sensors [26] to investigate how the reduction of noise and improvement of resolution in Depth could help to further improve the classification results. More advanced stages of development yet still accessible from the top view, could be investigated without targeting 3D reconstruction [55]. An issue comes with the possible overlapping between plants. One solution would be to decrease the density of plants but this would come with a lower throughput for the experiments. Another solution would be to investigate the possibility to track leaves during their growth in order to decipher partial occlusions. Here again, RGB depth sensors coupled with advanced machine learning approaches could be tested to further extend the capability to monitor seedling growth [56]. Last but not least, we can now directly apply the developed algorithms to analyze biologically in detail the statistical distribution of seedling growth events at night 
on large datasets. This may unravel new knowledge on the physiological impact of light on these growth kinetics in addition to their links with circadian rhythms [57].

\section{Conclusions}

In this article, we have demonstrated the added value of Depth when fused with RGB images for the important problem of detection of seedling growth stage development. During day time, Depth was shown to improve by $5 \%$ the classification performances on average. Also Depth was shown of value to refine the estimation of switch of growth stage during the night period. These results were established on different fusion strategies including CNN, TD-CNN-GRU and transformers. These methods were compared in order to incorporate the prior information of the order in which the different stages of the development occur. The best classification performance on these types of images was found with our optimized $\mathrm{CNN}$, which achieved $94 \%$ accuracy of detection. In our experiments all models and fusion strategies were trained and tested on several genotypes of beans.

Author Contributions: Conceptualization, D.R., P.R. and H.G.; methodology, D.R. and P.R.; software, H.G.; validation, D.R., P.R. and H.G.; formal analysis, H.G.; investigation, H.G.; resources, P.R.; data curation, H.G.; writing-original draft preparation, P.R.; writing-review and editing, D.R.; visualization, H.G.; supervision, D.R.; project administration, D.R.; funding acquisition, D.R. All authors have read and agreed to the published version of the manuscript.

Funding: This work is supported by PPR SUCSEED project, RFI OSMOSE project and by the Horizon 2020 Framework Program of the European Union under grant agreement No 817970 (INVITE: https:/ / www.h2020-invite.eu/, accessed on 10 December 2021).

Data Availability Statement: Data are available upon reasonable request.

Acknowledgments: The authors thank Marie Simonin, Arthur Coste from INRAe Angers for management of plants and Daniel Sochard, Remi Gardet for management of growth chamber part of PHENOTIC platform node of the french phenotyping national infrastructure PHENOME.

Conflicts of Interest: The authors declare no conflict of interest.

\section{References}

1. McCormac, A.C.; Keefe, P.D.; Draper, S.R. Automated vigour testing of field vegetables using image analysis. Seed Sci. Technol. 1990, 18, 103-112.

2. Sako, Y.; McDonald, M.B.; Fujimura, K.; Evans, A.F.; Bennett, M.A. A system for automated seed vigour assessment. Seed Sci. Technol. 2001, 29, 625-636.

3. Hoffmaster, A.L.; Fujimura, K.; McDonald, M.B.; Bennett, M.A. An automated system for vigor testing three-day-old soybean seedlings. Seed Sci. Technol. 2003, 31, 701-713. [CrossRef]

4. Marcos-Filho, J.; Bennett, M.; McDonald, M.; Evans, A.; Grassbaugh, E. Assessment of melon seed vigour by an automated computer imaging system compared to traditional procedures. Seed Sci. Technol. 2006, 34, 485-497. [CrossRef]

5. Marcos Filho, J.; Kikuti, A.L.P.; de Lima, L.B. Procedures for evaluation of soybean seed vigor, including an automated computer imaging system. Rev. Bras. Sementes 2009, 31, 102-112. [CrossRef]

6. Joosen, R.V.L.; Kodde, J.; Willems, L.A.J.; Ligterink, W.; van der Plas, L.H.W.; Hilhorst, H.W. germinator: A software package for high-throughput scoring and curve fitting of Arabidopsis seed germination. Plant J. 2010, 62, 148-159. [CrossRef] [PubMed]

7. Belin, É.; Rousseau, D.; Rojas-Varela, J.; Demilly, D.; Wagner, M.H.; Cathala, M.H.; Dürr, C. Thermography as non invasive functional imaging for monitoring seedling growth. Comput. Electron. Agric. 2011, 79, 236-240. [CrossRef]

8. Benoit, L.; Belin, É.; Dürr, C.; Chapeau-Blondeau, F.; Demilly, D.; Ducournau, S.; Rousseau, D. Computer vision under inactinic light for hypocotyl-radicle separation with a generic gravitropism-based criterion. Comput. Electron. Agric. 2015, $111,12-17$. [CrossRef]

9. Marcos Filho, J. Seed vigor testing: An overview of the past, present and future perspective. Sci. Agric. 2015, 72, 363-374. [CrossRef]

10. Gnädinger, F.; Schmidhalter, U. Digital counts of maize plants by unmanned aerial vehicles (UAVs). Remote Sens. 2017, 9, 544. [CrossRef]

11. Sadeghi-Tehran, P.; Sabermanesh, K.; Virlet, N.; Hawkesford, M.J. Automated method to determine two critical growth stages of wheat: Heading and flowering. Front. Plant Sci. 2017, 8, 252. [CrossRef] [PubMed]

12. Rasti, P.; Demilly, D.; Benoit, L.; Belin, E.; Ducournau, S.; Chapeau-Blondeau, F.; Rousseau, D. Low-cost vision machine for high-throughput automated monitoring of heterotrophic seedling growth on wet paper support. In Proceedings of the British Machine Vision Conference 2018, BMVC 2018, Newcastle, UK, 3-6 September 2018; p. 323. 
13. Chen, R.; Chu, T.; Landivar, J.A.; Yang, C.; Maeda, M.M. Monitoring cotton (Gossypium hirsutum L.) germination using ultrahigh-resolution UAS images. Precis. Agric. 2018, 19, 161-177. [CrossRef]

14. Zhao, B.; Zhang, J.; Yang, C.; Zhou, G.; Ding, Y.; Shi, Y.; Zhang, D.; Xie, J.; Liao, Q. Rapeseed seedling stand counting and seeding performance evaluation at two early growth stages based on unmanned aerial vehicle imagery. Front. Plant Sci. 2018, 9, 1362. [CrossRef]

15. Jiang, Y.; Li, C.; Paterson, A.H.; Robertson, J.S. DeepSeedling: Deep convolutional network and Kalman filter for plant seedling detection and counting in the field. Plant Methods 2019, 15, 141. [CrossRef] [PubMed]

16. Samiei, S.; Rasti, P.; Vu, J.L.; Buitink, J.; Rousseau, D. Deep learning-based detection of seedling development. Plant Methods 2020, 16, 103. [CrossRef]

17. Chéné, Y.; Rousseau, D.; Lucidarme, P.; Bertheloot, J.; Caffier, V.; Morel, P.; Belin, É.; Chapeau-Blondeau, F. On the use of depth camera for 3D phenotyping of entire plants. Comput. Electron. Agric. 2012, 82, 122-127. [CrossRef]

18. Nock, C.; Taugourdeau, O.; Delagrange, S.; Messier, C. Assessing the potential of low-cost 3D cameras for the rapid measurement of plant woody structure. Sensors 2013, 13, 16216-16233. [CrossRef] [PubMed]

19. Paulus, S.; Behmann, J.; Mahlein, A.K.; Plümer, L.; Kuhlmann, H. Low-cost 3D systems: Suitable tools for plant phenotyping. Sensors 2014, 14, 3001-3018. [CrossRef] [PubMed]

20. Rousseau, D.; Chéné, Y.; Belin, E.; Semaan, G.; Trigui, G.; Boudehri, K.; Franconi, F.; Chapeau-Blondeau, F. Multiscale imaging of plants: Current approaches and challenges. Plant Methods 2015, 11, 6. [CrossRef]

21. Rosell-Polo, J.R.; Gregorio, E.; Gené, J.; Llorens, J.; Torrent, X.; Arnó, J.; Escola, A. Kinect v2 sensor-based mobile terrestrial laser scanner for agricultural outdoor applications. IEEE/ASME Trans. Mechatron. 2017, 22, 2420-2427. [CrossRef]

22. Vit, A.; Shani, G. Comparing rgb-d sensors for close range outdoor agricultural phenotyping. Sensors 2018, 18, 4413. [CrossRef] [PubMed]

23. Perez, R.M.; Cheein, F.A.; Rosell-Polo, J.R. Flexible system of multiple RGB-D sensors for measuring and classifying fruits in agri-food Industry. Comput. Electron. Agric. 2017, 139, 231-242. [CrossRef]

24. Martinez-Guanter, J.; Ribeiro, Á.; Peteinatos, G.G.; Pérez-Ruiz, M.; Gerhards, R.; Bengochea-Guevara, J.M.; Machleb, J.; Andújar, D. Low-cost three-dimensional modeling of crop plants. Sensors 2019, 19, 2883. [CrossRef]

25. Reynolds, D.; Baret, F.; Welcker, C.; Bostrom, A.; Ball, J.; Cellini, F.; Lorence, A.; Chawade, A.; Khafif, M.; Noshita, K.; et al. What is cost-efficient phenotyping? Optimizing costs for different scenarios. Plant Sci. 2019, 282, 14-22. [CrossRef] [PubMed]

26. Servi, M.; Mussi, E.; Profili, A.; Furferi, R.; Volpe, Y.; Governi, L.; Buonamici, F. Metrological Characterization and Comparison of D415, D455, L515 RealSense Devices in the Close Range. Sensors 2021, 21, 7770. [CrossRef] [PubMed]

27. Baltrušaitis, T.; Ahuja, C.; Morency, L.P. Multimodal machine learning: A survey and taxonomy. IEEE Trans. Pattern Anal. Mach. Intell. 2018, 41, 423-443. [CrossRef] [PubMed]

28. Atrey, P.K.; Hossain, M.A.; El Saddik, A.; Kankanhalli, M.S. Multimodal fusion for multimedia analysis: A survey. Multimed. Syst. 2010, 16, 345-379. [CrossRef]

29. Ramachandram, D.; Taylor, G.W. Deep multimodal learning: A survey on recent advances and trends. IEEE Signal Process. Mag. 2017, 34, 96-108. [CrossRef]

30. Valada, A.; Oliveira, G.L.; Brox, T.; Burgard, W. Deep multispectral semantic scene understanding of forested environments using multimodal fusion. In Proceedings of the International Symposium on Experimental Robotics, Nagasaki, Japan, 3-8 October 2016.

31. Andreas, E.; Jost, T.S.; Luciano, S.; Martin, R.; Wolfram, B. Multimodal deep learning for robust RGB-D object recognition. In Proceedings of the International Conference on Intelligent Robots and Systems (IROS), Hamburg, Germany, 28 September-2 October 2015.

32. Jordi, S.R.; Kai-Lung, H.; Yuan-Sheng, H.; Tekoing, L.; Shintami, C.; Wen-Huang, C. A comparative study of data fusion for RGB-D based visual recognition. Pattern Recognit. Lett. 2016, 73, 1-6.

33. Wang, A.; Lu, J.; Cai, J.; Cham, T.J.; Wang, G. Large-margin multimodal deep learning for RGB-D object recognition. IEEE Trans. Multimed. 2015, 17, 1887-1898.

34. Bezen, R.; Edan, Y.; Halachmi, I. Computer vision system for measuring individual cow feed intake using RGB-D camera and deep learning algorithms. Comput. Electron. Agric. 2020, 172, 105345.

35. Srivastava, N.; Salakhutdinov, R. Learning representations for multimodal data with deep belief nets. In Proceedings of the 29th International Conference Machine Learning (Workshop), Edinburgh, UK, 26 June-1 July 2012.

36. Yu, C.; Shawn, S.; Jianbiao, H.; Degui, X.; Cui, T.; Ping, C.; Henning, M. Medical image retrieval: A multimodal approach. Cancer Inform. 2014, 13, 125.

37. Lenz, I.; Lee, H.; Saxena, A. Deep learning for detecting robotic grasps. Int. J. Robot. Res. 2015, 34, 705-724.

38. Ashesh, J.; Avi, S.; Hema, S.K.; Shane, S.; Ashutosh, S. Recurrent neural networks for driver activity anticipation via sensory-fusion architecture. In Proceedings of the 2016 IEEE International Conference on Robotics and Automation (ICRA), Stockholm, Sweden, 16-21 May 2016.

39. Xinhang, S.; Shuqiang, J.; Luis, H.; Chengpeng, C. Learning effective RGB-D representations for scene recognition. IEEE Trans. Image Process. 2019, 28, 980-993. 
40. Cheng, Y.; Zhao, X.; Cai, R.; Li, Z.; Huang, K.; Rui, Y. Semi-supervised multimodal deep learning for RGB-D object recognition. In Proceedings of the Twenty-Fifth International Joint Conference on Artificial Intelligence (IJCAI-16), New York, NY, USA, 9-15 July 2016.

41. Li, S.; Cheng, Z.; Rustam, S. Weakly-supervised DCNN for RGB-D object recognition in real-world applications which lack large-scale annotated training data. arXiv 2017, arXiv:1703.06370.

42. Graves, A.; Mohamed, A.r.; Hinton, G. Speech recognition with deep recurrent neural networks. In Proceedings of the 2013 IEEE International Conference on Acoustics, Speech and Signal Processing, Vancouver, BC, Canada, 26-31 May 2013; pp. 6645-6649.

43. Garbouge, H.; Rasti, P.; Rousseau, D. Deep learning-based detection of seedling development from indoor to outdoor. In Proceedings of the International Conference on Systems, Signals and Image Processing (IWSSIP), Bratislava, Slovakia, 2-4 June 2021; Volume 1, pp. 1-11.

44. Vaswani, A.; Shazeer, N.; Parmar, N.; Uszkoreit, J.; Jones, L.; Gomez, A.N.; Kaiser, L.; Polosukhin, I. Attention is all you need. arXiv 2017, arXiv:1706.03762.

45. Minervini, M.; Giuffrida, M.V.; Perata, P.; Tsaftaris, S.A. Phenotiki: An open software and hardware platform for affordable and easy image-based phenotyping of rosette-shaped plants. Plant J. 2017, 90, 204-216. [CrossRef]

46. Intel RealSense Documentation-Intel RealSense Depth Tracking Cameras. Available online: https://dev.intelrealsense.com/ docs / docs-get-started (accessed on 7 December 2019).

47. Granados, M.; In-Kim, K.; Tompkin, J.; Kautz, J.; Theobalt, C. Background Inpainting for Videos with Dynamic Objects and a Free-moving Camera. In Proceedings of the European Conference on Computer Vision (ECCV), Florence, Italy, 7-13 October 2012.

48. Couprie, C.; Farabet, C.; Najman, L.; LeCun, Y. Indoor semantic segmentation using depth information. arXiv 2013, arXiv:1301.3572.

49. Krizhevsky, A.; Sutskever, I.; Hinton, G.E. Imagenet classification with deep convolutional neural networks. Adv. Neural Inf. Process. Syst. 2012, 25, 1097-1105. [CrossRef]

50. Yin, W.; Kann, K.; Yu, M.; Schütze, H. Comparative study of CNN and RNN for natural language processing. arXiv 2017, arXiv:1702.01923.

51. Zhou, K.; Wang, W.; Hu, T.; Deng, K. Time Series Forecasting and Classification Models Based on Recurrent with Attention Mechanism and Generative Adversarial Networks. Sensors 2020, 20, 7211. [CrossRef]

52. Yuan, Y.; Lin, L. Self-Supervised Pre-Training of Transformers for Satellite Image Time Series Classification. IEEE J. Sel. Top. Appl. Earth Obs. Remote Sens. 2020, 14, 474-487. [CrossRef]

53. Garnot, V.S.F.; Landrieu, L.; Giordano, S.; Chehata, N. Satellite image time series classification with pixel-set encoders and temporal self-attention. In Proceedings of the IEEE/CVF Conference on Computer Vision and Pattern Recognition, Seattle, WA, USA, 13-19 June 2020; pp. 12325-12334.

54. Dosovitskiy, A.; Beyer, L.; Kolesnikov, A.; Weissenborn, D.; Zhai, X.; Unterthiner, T.; Dehghani, M.; Minderer, M.; Heigold, G.; Gelly, S.; et al. An image is worth 16x16 words: Transformers for image recognition at scale. arXiv 2020, arXiv:2010.11929.

55. Sampaio, G.S.; Silva, L.A.d.; Marengoni, M. 3D Reconstruction of Non-Rigid Plants and Sensor Data Fusion for Agriculture Phenotyping. Sensors 2021, 21, 4115. [CrossRef] [PubMed]

56. Jin, J.; Dundar, A.; Bates, J.; Farabet, C.; Culurciello, E. Tracking with deep neural networks. In Proceedings of the 2013 47th Annual Conference on Information Sciences and Systems (CISS), Baltimore, MD, USA, 20-22 March 2013; pp. 1-5.

57. Srivastava, D.; Shamim, M.; Kumar, M.; Mishra, A.; Maurya, R.; Sharma, D.; Pandey, P.; Singh, K. Role of circadian rhythm in plant system: An update from development to stress response. Environ. Exp. Bot. 2019, 162, 256-271. [CrossRef] 